https://creativecommons.org/licenses/by/4.0/

\title{
RECORRIDO HISTÓRICO CENTRO COMERCIAL PLAZA DE MERCADO DE UBATÉ
}

\author{
Historical route plaza de mercado de Ubaté shopping center
}

NORMA C NARVÁEZ ${ }^{1}$, NUBIA M GUERRERO², GINA Y. FUENTES ${ }^{3}$

Recibido:19 de junio de 2021. Aceptado:19 de julio de 2021

DOI: http://dx.doi.org/10.21017/rimci.2021.v8.n16.a100

\begin{abstract}
Resumen
La Villa de San Diego de Ubaté ha sido la cabecera principal municipal, siendo así el centro de comercio de mayor afluencia, reuniendo a campesinos no sólo de sabana centro, sino de Bogotá también, personas que se reúnen en torno a tradiciones y venta de productos que son cosechados por sí mismos y por sus familias, siendo así el sustento familiar. El objetivo del presente estudio es realizar un diagnostico de los trabajadores informales que laboran en la Plaza de Mercado Villa de san Diego de Ubaté. La presente investigación es un estudio observacional, de corte transversal, de enfoque cuantitativo y alcance descriptivo.

Se diseñó un instrumento que evalúa variables sociodemográficas, ocupacionales, estado de salud y condiciones de seguridad y salud en el trabajo, la muestra fue de 183 puestos de trabajo quienes participaron de manera voluntaria. Los hallazgos principales se encuentran en el número de personas que laboran especialmente en las secciones de batán (43\%), campesinos (25\%) y fruver (15\%), evidenciando molestias a nivel osteomuscular principalmente. Se concluye que se hace necesario la implementación de medidas de control en la persona, en la fuente y en el medio, con el fin de mejorar las condiciones de seguridad y salud en el trabajo de los trabajadores informales.
\end{abstract}

Palabras clave. Cultura; plaza de mercado; trabajador informal; trabajo informal; tradición.

\begin{abstract}
Some The Villa of San Diego de Ubaté has been the main municipal seat, thus being the commercial center with the greatest influx, bringing together peasants not only from the central savannah, but also from Bogotá, people who gather around traditions and sale of products. that are harvested by themselves and by their families, thus being the family sustenance. The objective of this study is to make a diagnosis of informal workers who work in the Plaza de Mercado Villa de San Diego de Ubaté. This research is an observational, cross-sectional study with a quantitative approach and descriptive scope.

An instrument was designed that assesses sociodemographic and occupational variables, health status, and health and safety conditions at work, the sample was 183 jobs who participated voluntarily. The main findings are found in the number of people who work especially in the batán (43\%), peasant (25\%) and fruver (15\%) sections, showing discomfort mainly at the musculoskeletal level. It is concluded that it is necessary to implement control measures in the person, at the source and in the middle, in order to improve the health and safety conditions at work of informal workers.
\end{abstract}

Keywords. Culture, market square, informal worker, informal work, tradition.

1 Facultad de ciencia empresariales, Egresado Programa de Administración en Seguridad y Salud en el Trabajo, Corporación Universitaria Minuto de Dios - UNIMINUTO, Colombia. Correo electrónico: nnarvaezcor@uniminuto.edu.co

2 Facultad de ciencia empresariales, Egresado Programa de Administración en Seguridad y Salud en el Trabajo, Corporación Universitaria Minuto de Dios - UNIMINUTO, Colombia. Correo electrónico: nguerreroca@uniminuto.edu.co

3 Facultad de ciencia empresariales, Programa de Administración en Seguridad y Salud en el Trabajo, Corporación Universitaria Minuto de Dios UNIMINUTO, Colombia. Correo electrónico: gina.fuentes@uniminuto.edu.co 


\section{INTRODUCCIÓN}

L AS PLAZAS de mercado por tradición han sido lugares que se han centrado en vender productos cosechados por los campesinos, encontrando víveres, alimentos de primera necesidad incluidos utensilios de aseo. Un aspecto interesante que caracteriza a las plazas de mercado, es el encontrar alimentos, vestuario, muebles, animales, artesanías entre otros.

La plaza de mercado Villa de San Diego de Ubaté, inició en el parque Juan José Neira alrededor de los años 70, esta plaza fue tradicionalmente conocida para todos los transeuntes quienes durante su recorrido visitaban Ubaté, continuando su camino hacia Boyacá e incluso Santander; fue conocida por sus grandes sabores, por la delicadeza y especial atención de los campesinos y en especial por encontrar todo en un mismo lugar; sin embargo, la plaza de mercado fue creciendo y con el objetivo de organizar a las personas que cada día iban en aumento, se tomó la decisión de organizar la plaza de mercado en un espacio más amplio distribuido en 4 sectores: fruver, papa, comida y batán, de tal manera que las personas pudieran identificar sus necesidades y pudieran realizar un desplazamiento más seguro en búsqueda de realizar sus compras y encontrar sus productos. Esta reubicación se realizó en el año 2002 [1].

Con el paso del tiempo y la organización presentada, se empezó a identificar que estos lugares estaban presentes no solo en los pueblos sino en las ciudades, generando un gran número de trabajadores "informales", considerando que no eran contratados por medio de un contrato laboral con cobertura de un sistema de salud; adicionalmente, se encontraba otro agravante relacionado con el número de horas que permanecían trabajando en la plaza de mercado, teniendo en cuenta que los comerciantes y productores eran los mismos vendedores; por lo tanto, es evidente encontrar familias completas quienes han optado por continuar con la tradición familiar y evidentemente sin protección laboral alguna [2].

Las plazas de mercado son patrimonio cultural, por lo tanto, se busca la preservación y mantenimiento de las mismas; de hecho, para Martinez de Rojas, hace referencia que las plazas de mercado siendo lugares de intercambio cultural a nivel intergeneracional, deben ser diagnosticadas, evaluadas e intervenidas, con el ánimo de mejorar no solo sus condiciones físicas, sino ocupacionales [3]. Un aspecto importante a tener presente son las condiciones de seguridad y salud en el trabajo, teniendo en cuenta la prevalencia de riesgos que se pueden generar con ocasión del trabajo que desarrollan en las plazas de mercado. La identificación y control de riesgos es un aspecto relevante para implementar con los trabajadores de la plaza de mercado, en dónde prevalece la informalidad, la desprotección en salud, pensión y aseguradora de riesgos laborales, cuyas actividades pueden generar accidentes de trabajo y se convierte en un factor de riesgo permanente, cuya implementación normativa es una necesidad apremiante con un grupo poblacional que crece cada día más, pero que a nivel de protección decrece. Agencia de Investigación de Seguridad y Salud en el Trabajo [4].

\section{Materiales Y MÉtodos}

Tipo de estudio: La presente investigación es observacional de corte transversal, enfoque cuantitativo de alcance descriptivo. La población objetivo son todos lo trabajadores de la plaza de mercado; sin embargo para determinar la muestra se utilizó un muestreo por conveniencia estableciendo así 183 puestos de trabajo objeto de estudio; es importante aclarar que se habla de puestos de trabajo, ya que hay propietarios de 2 y 3 puestos atendidos por personas diferentes. Se diseñó un instrumento para la recolección de la información: con las siguientes variables: Sociodemográficas, hábitos saludables, condiciones de salud, condiciones laborales, y condiciones de seguridad en el trabajo. Es así cómo se pudo realizar un acercamiento con los trabajadores en sus puestos de trabajo, realizando algunas preguntas claves para identificar mejor los riesgos presentes.

\section{Resultados}

A continuación se reflexionará sobre los aspectos más relevantes relacionados con la cultura, seguridad y salud en el trabajo y la historia de la plaza de mercado Villa de San Diego de Ubaté. En donde los principales aportes se han generado desde los trabajadores informales y sobre las condiciones principales de seguridad y salud en

Rev. Ingeniería, Matemáticas y Ciencias de la Información Vol. 8 / Núm. 16 / julio - diciembre de 2021; pág. 37-42 
el trabajo en el cual se enfrentan las personas al realizar sus trabajos desde una perspectiva de informalidad.

Tabla I. Distribución porcentual de los trabajadores por secciones en la plaza de mercado.

\begin{tabular}{|c|c|c|}
\hline Sector Trabajador & N & $\%$ \\
\hline Restaurantes & 21 & $11 \%$ \\
\hline Administración & 0 & $0 \%$ \\
\hline Cárnicos & 3 & $2 \%$ \\
\hline Hierbas y Fruver & 27 & $15 \%$ \\
\hline Pescado y queso & 0 & $0 \%$ \\
\hline Batán & 79 & $43 \%$ \\
\hline Baños Mayorista & 0 & $0 \%$ \\
\hline Campesinos & 46 & $25 \%$ \\
\hline otros & 7 & $4 \%$ \\
\hline Total & $\mathbf{1 8 3}$ & $\mathbf{1 0 0} \%$ \\
\hline
\end{tabular}

Fuente: Elaboración propia.

Un aspecto relevante a observar en esta tabla es que en un $43 \%$ de los trabajadores se encuentran en Batán (vestuario y calzado), seguido de un $25 \%$ correspondiente a campesinos y un $15 \%$ en hierbas y fruver principalmente.

Tabla II. Distribución porcentual de afiliación a ARL de los trabajadores

\begin{tabular}{|c|c|c|}
\hline Afiliación ARL & $\mathbf{n}$ & $\%$ \\
\hline $\mathrm{Si}$ & 5 & $3 \%$ \\
\hline No & 178 & $97 \%$ \\
\hline NR & 0 & $0 \%$ \\
\hline Total & $\mathbf{1 8 3}$ & $\mathbf{1 0 0} \%$ \\
\hline
\end{tabular}

Fuente: Elaboración propia.

Un aspecto importante se encuentra en la afiliación al sistema de seguridad social, en dónde en un $80 \%$ corresponden al régimen subsidiado y tan solo el $20 \%$ a contributivo, y de este $20 \%$ tan solo un 3\% están afiliados a la aseguradora de Riesgos laborales, situación preocupante, si tenemos en cuenta que el tipo de trabajo que realizan las personas es un trabajo de manipulación de cargas manuales y posturas mantenidas y prolongadas la mayor parte del tiempo.
Tabla III. Distribución porcentual del estado civil de los trabajadores.

\begin{tabular}{|c|c|c|}
\hline Estado Civil & $\mathbf{n}$ & $\%$ \\
\hline Casado & 47 & $26 \%$ \\
\hline Soltero & 53 & $29 \%$ \\
\hline Unión Libre & 59 & $32 \%$ \\
\hline Viudo & 10 & $5 \%$ \\
\hline Separado & 14 & $8 \%$ \\
\hline NR & & $0 \%$ \\
\hline Total & $\mathbf{1 8 3}$ & $\mathbf{1 0 0} \%$ \\
\hline
\end{tabular}

Fuente: Elaboración propia.

En cuanto al estado civil se observa porcentajes similares en el género casado $(26 \%)$, soltero $(29 \%)$ y unión libre (32).

Tabla IV. Distribución porcentual del número de personas a cargo.

\begin{tabular}{|c|c|c|}
\hline Personas a Cargo & n & $\%$ \\
\hline Ninguna & 48 & $26 \%$ \\
\hline 1 a 3 & 108 & $59 \%$ \\
\hline 4 a 6 & 25 & $14 \%$ \\
\hline Más de 6 & 2 & $1 \%$ \\
\hline NR & 0 & $0 \%$ \\
\hline Total & $\mathbf{1 8 3}$ & $\mathbf{1 0 0} \%$ \\
\hline
\end{tabular}

Fuente: Elaboración propia.

Respecto al número de personas a cargo, es importante tener presente que el $74 \%$ de la población tienen personas a cargos con mayor prevalencia entre 1 a 3 personas; de igual manera, es importante tener presente que el género femenino está presente en un $77 \%$, lo que indica que al terminar las actividades laborales en la plaza de mercado deben llegar a sus hogares a realizar actividades adicionales que pueden influir en su estado de salud.

Tabla V. Distribución porcentual del uso de los EPP.

\begin{tabular}{|c|c|c|}
\hline ¿Utiliza EPI? & $\mathbf{n}$ & $\%$ \\
\hline $\mathrm{Si}$ & 110 & $60 \%$ \\
\hline No & 73 & $40 \%$ \\
\hline $\mathrm{NR}$ & 0 & $0 \%$ \\
\hline Total & $\mathbf{1 8 3}$ & $\mathbf{1 0 0} \%$ \\
\hline
\end{tabular}

Fuente: Elaboración propia. 
El uso de Elementos de protección personal está presente en un $60 \%$, sin embargo, al evaluar los tipos de EPP que utilizan, se observa que son más relevantes en la sección de comidas, pero para el caso de levantamiento de cargas no es frecuente.

Tabla Vl. Distribución porcentual de la práctica de deportes.

\begin{tabular}{|c|c|c|}
\hline ¿Práctica algún Deporte? & $\mathbf{n}$ & $\%$ \\
\hline $\mathrm{Si}$ & 40 & $22 \%$ \\
\hline No & 143 & $78 \%$ \\
\hline NR & 0 & $0 \%$ \\
\hline Total & $\mathbf{1 8 3}$ & $\mathbf{1 0 0} \%$ \\
\hline
\end{tabular}

Fuente: Elaboración propia

Del total de la muestra del estudio, un 22\% realiza algún tipo de ejercicio, entendiéndolo como una actividad por más de 30 minutos

Tabla VII. Distribución porcentual de presencia de sintomas durante el último mes.

\begin{tabular}{|c|c|c|}
\hline Síntomas en el Último Mes & $\mathbf{n}$ & $\%$ \\
\hline Dolor de Cabeza & 87 & $19 \%$ \\
\hline Dolor de Cuello & 41 & $9 \%$ \\
\hline Dolor de Brazos & 52 & $12 \%$ \\
\hline Dolor de Manos & 57 & $13 \%$ \\
\hline Adormecimiento en Manos & 27 & $6 \%$ \\
\hline Dolor de Espalda & 56 & $12 \%$ \\
\hline Dolor de Piernas & 42 & $9 \%$ \\
\hline Adormecimiento Piernas & 25 & $6 \%$ \\
\hline Tos Frecuente & 9 & $2 \%$ \\
\hline Gripa frecuente & 15 & $3 \%$ \\
\hline Alergias & 10 & $2 \%$ \\
\hline Dolor de Estómago & 5 & $1 \%$ \\
\hline Sentimientos de Tristeza & 24 & $5 \%$ \\
\hline Total & $\mathbf{4 5 0}$ & $\mathbf{1 0 0} \%$ \\
\hline
\end{tabular}

Fuente: Elaboración propia.

La presencia de sintomatología asociada al trabajo es relevante, ya que en un 19\% se encuentra la presencia de dolor de cabeza, seguido de dolor de manos $(13 \%)$ y dolor de brazos $(12 \%)$, en menor proporción se encuentran otras dolencias relacionadas tanto con miembros superiores como con miembros inferiores; la presencia de esta sintomatología se observa en un mismo trabajador de 2 a 3 síntomas.

\section{ANÁlisis de LA INFORMación}

Es interesante los resultados iniciales del diagnostico de la información recolectada con los trabajadores de la plaza de mercado de la Villa de san Diego de Ubaté; en este orden de ideas, lo que hoy en día se conoce como plaza de mercado como un lugar de tradición y cultura proveniente de los campesinos de la zona, convirtiéndose en un lugar de intercambio de saberes y reencuentro de personas. En este sentido es relevante traer a colación algunos aspectos que han marcado la historia de la plaza de mercado para así entender su relación con los hallazgos del estudio.

Hacia el siglo XVI cuando se presentó la colonización con la llegada de los españoles, se empezó un fenómeno de migración interesante hacia diferentes territorios, lugares otorgados para los indígenas, y desde este momento se observaban los sectores de agricultura y de artesanos; de acuerdo a la gran variedad de productos que se ofrecían se realizaba un intercambio de productos interesante, un lugar denominado la Plaza Mayor, muy similar a lo que vemos hoy en día. Es así cómo las plazas mayores llegaron a convertirse en las plazas de mercado, lugares caracterizados por la venta de productos cultivados por los campesinos de las diferentes regiones. Un dato interesante radica en la diferencia que se puede encontrar en estos lugares tradicionales, ya que dependiendo del municipio se realizan una serie de festividades que enmarcan la tradición y cultura de ese municipio, convirtiendo cada lugar como un escenario tradicional que enmarca a los residentes de cada región, claro está que son bienvenidos campesinos de otros lugares, siempre que se acomoden a las condiciones de cada municipio [5].

Siendo las plazas de mercado un lugar de abastecimiento no solo de productos, sino de intercambio de saberes, es necesario enmarcar el pilar de los aspectos culturales y sociales que se tejen en torno a las plazas de mercado; realmente, se enmarcan en una trascendencia familiar, en donde estas costumbres son heredadas de generación en generación, muchos adolescentes con estas herencias buscan un cambio de tradiciones, generalmente buscan "mejores condiciones y status", olvidando que esta tradición y cultura se debe conservar con el fin de mantener esta tradición y evitar que lleguen a extinguirse; pese a los cambios que han

Rev. Ingeniería, Matemáticas y Ciencias de la Información Vol. 8 / Núm. 16 / julio - diciembre de 2021; pág. 37-42 
traido las urbes respecto a "placitas y fruver" que ofrecen productos en diferentes presentaciones, empacados en bolsas y presentados de manera brillante siendo atractivos a la vista, las plazas tradicionales siguen siendo de preferencia no solo para el campesino sino para el "citadino", en dónde se encuentra, ese valor agregado, conocido popularmente como "el encime" el cuál se presenta de manera permanente en las plazas de mercado, ya que en los fruver actuales el costo es generado por el valor exacto del producto adquirido. Es así, cómo asistir a una plaza de mercado es regresar a las tradiciones y costumbres de nuestros antepasados y preservar el contacto con nuestros campesinos [2].

El epicentro de la plaza de mercado de Villa de San Diego de Ubaté inició frente a la Estación de Policía en el Parque Juan José Neira, se encontraba de todos los productos que fueran de necesidad abarcando zapatos, muebles y por supuesto comi$\mathrm{da}$, incluyendo las verduras y las frutas, evidentemente se observaba desorden, riesgos y no se evidenciaba un ente de control para el manejo de esta gran plaza mayor. De hecho, los espacios no estaban acondicionados para la comercialización de los diferentes productos, se veían obligados a instalar casetas con lonas con el fin de evitar un poco el exceso de lluvia y sol [2]. Posteriormente, fueron trasladados a la plaza Ricaurte, un espacio de tradición conocido por las casetas turísticas; en este lugar debían pagar una suma al municipio de acuerdo a los días que abrieran al público; sin embargo, era un espacio provisional, pues al ser un número considerable de campesinos que ofrecían sus productos, se veía la necesidad de generar un lugar más amplio, cómodo y adecaudo para la venta de diferentes productos considerando tradicionalmente el día de mercado.

Hacia el año 2002, se realizó la reubicación de la plaza de mercado, realizando una edificación con 4 bodegas, separadas por sección A: papa y granos, sección B: hierbas y fruver, sección C: batán y bogotanos y sección D: comida, incluyendo cárnicos y licogret. sin embargo, aunque se ve más ordenado esta reubicación solo fue pensada en mejorar el orden y dividir los productos, pero nada relacionado con el control de accidentalidad o condiciones mínimos para cumplir las labores a los campesinos de la zona. Existe un administrador de la plaza, quién se encarga de la comunicación permanente entre la secretaría de gobierno y los campesinos y quienes realizan un acercamiento con entidades educativas con el fin de realizar estudios para mejorar las condiciones de los mismos y así poder intervenir de manera impactante de acuerdo a las necesidades de la población y al cumplimiento normativo.

De acuerdo a Gómez \& Marroquín, algunos productos son traídos desde La Corporación de Abastos de Bogotá (Corabastos) y otros de los municipios aledaños de acuerdo a las cosechas presentes y a los productos ofertados [6]; es así cómo se busca mantener la tradición en un espacio más amplio y con mejores condiciones físicas mejores que en los antiguos lugares. Sin embargo, es importante resaltar que no basta la reubicación, que es necesario continuar con mejoras físicas, aunque cada propietario de cada espacio es libre de adecuar su espacio y convertirlo en un lugar más llamativo no es suficiente, ya que se hace necesario realizar estudios e intervenir a la población en materia de seguridad y salud en el trabajo.

Con base en los resultados de este diagnostico inicial, se logra observar que los trabajadores informales se encuentran expuestos a peligros físicos, ergonómicos, biológicos, psicosociales y públicos, de hecho, el riesgo es prevalente y el desconocimiento del significado de peligro o riesgo, o a cuáles riesgos están expuestos, genera que la población esté de manera permanente enfrentada a accidentes laborales o al inicio de enfermedades de tipo laboral; sin embargo, preocupa más el observar que existe un porcentaje muy bajo de personas que pertenecen al régimen contributivo, ya que al ser denominados como trabajadores informales, no ven la necesidad de cotizar a la aseguradora de riesgos laborales, empresas prestadoras de salud y pensiones. Otro aspecto importante, que se analiza es la carencia de plan de emergencias y programa de capacitaciones generando una necesidad inmediata de intervención y de concientizar a la población informal sobre la necesidad de cotizar a un sistema general de salud, que estén protegidos y puedan realizar acciones para evitar accidentes y enfermedades que pueden afectar de manera importante la funcionalidad y recursos económicos de la población trabajadora.

Aunque en la actualidad existe la ley 1988 del 2 de agosto de 2019 "por la cual se establecen los 
lineamientos, para la formulación, implementación y evaluación de una política pública de los vendedores informales y se dictan otras disposiciones" [7], aún sigue prevaleciendo este tipo de labor, sigue siendo un proceso que requiere de especial atención para poder lograr un cambio y generar la implementación de acciones acordes a las necesidades poblacionales [8]. Es así como se hace primordial poder implementar planes de acción que mejoren las condiciones laborales de la población informal [9].

\section{Conclusiones}

Se hace necesario intervenir a la población informal pertenecientes a las plazas de mercado de ubaté, implementando planes de acción que permitan mejorar sus condiciones laborales y se propicie espacios de capacitación que minimicen la probabilidad de ocurrencia de accidentes laborales e incluso el desarrollo de enfermedades laborales. En este orden de ideas, es importante preservar las plazas de mercado y generar consciencia del autocuidado, de tal forma que se generen mejores condiciones de vida tanto personales como laborales, dando cumplimiento a la normatividad actual aplicable.

Se hace necesario y prioritario continuar con estudios relacionados a la seguridad y salud en el trabajo, de tal manera que las acciones que se implementen mejoren las condiciones en salud de las personas que laboran de manera informal.

\section{REFERENCIAS}

[1] P. Escallón, HSB Noticias.com. Obtenido de https://hsbnoticias.com/noticias/economia/fotos-ubate-cuenta-con-uno-de-los-mejores-dentrosde-acopio-165730. 2019.

[2] K. Vargas, La Plaza de mercado. Periódico El Campesino. Obtenido de http:/ / www.elcampesino.co/ la-plaza-de-mercado-2/. 2015.

[3] A. Martinez de Rojas, Visión Ubaté. Ubaté. 1992.

[4] Agencia Internacional de Seguridad y Salud en el Trabajo. Occupational safety and health (OSH) in the informal economy (Ginebra). Disponible en: https://www.ilo.org/wcmsp5/groups/public/ - ed_protect/ - protrav/ - safework/documents/ publication/wcms_313828.pdf. 2014.

[5] F. Seño, Acerca del valor patrimonial de los mercados municipales y plazas de abastos de nuestro entorno. Cuadernos de los Amigos de los Museos de Osuna, 1-5. 2013.

[6] N. Gómez y K. Marroquín, Agricultura Urbana en el Municipio de Chía con Familias de Estratos Bajos. Extraído de: http:/ / repository.udca.edu.co: 8080/jspui/bitstream/11158/156/1/203505.pdf. 2011.

[7] Constitución política de Colombia. (1991).

[8] R. Ayala, De la Provincia de Ubaté. En Caracterización Económica y Empresarial (págs. 18-22). Bogotá. 2008.

[9] M. V. Rubio Pabón, El trabajo informal en Colombia e impacto en América Latina. Observatorio Laboral Revista Venezolana, 23-40. Obtenido de https:/ / www.redalyc.org/articulo.oa?id=2190/ 219030399002. 2014. 